\title{
The neoflavonoid latifolin isolated from Dalbergia odorifera attenuates hypoxia- reoxygenation injury by reactive oxygen species scavenging and Nrf2 activation in $\mathrm{H} 9 \mathrm{c2}$ cardiomyocyte
}

\author{
Lanying Chen ${ }^{1}$, Yao Luo ${ }^{1}$, Xueliang Li ${ }^{1}$, Ronghua Liư $^{2}$, Feng Shao ${ }^{2}$ \\ ${ }^{I}$ National Pharmaceutical Engineering Center for Solid Preparation in Chinese Herbal Medicine, Jiangxi University of \\ Traditinal Chinese Medicine, China, ${ }^{2}$ The School of Pharmacy, Jiangxi University of Traditional Chinese Medicine, \\ China
}

Latifolin, as a major neoflavonoid component isolated from the heartwood of Dalbergia odorifera T. Chen, possesses antioxidant, anti-inflammatory, vasodilatory properties, etc. This study aimed to explore the effects of latifolin on the hypoxia-reoxygenation $(\mathrm{H} / \mathrm{R})$ injury in $\mathrm{H} 9 \mathrm{c} 2$ cardiomyocytes and further investigate its possible molecular mechanisms. H9c2 cardiomyocytes pretreated with latifolin were exposed to hypoxia and reoxygenation. Cell viability and lactate dehydrogenase (LDH) activity in culture medium were determined by MTT and microcolorimetry. The expression and level of intracellular reactive oxygen species (ROS) were measured by DCFH-DA staining and flow cytometry. The expression and location of Nrf2 were identified by immunocytochemistry. The expression of Nrf2 and HO-1 were detected by Western blotting. The results showed that latifolin increased the cell viability, reduced LDH activity and ROS production. Immunocytochemistry and western blot analysis showed that latifolin increased the expression of Nrf2 both in cytoplasm and nucleus and HO-1 in cytoplasm. These findings indicated that the anti-oxidative and ROS scavenging activity of latifolin was related to activating Nrf2/ARE pathway, which can attenuate $\mathrm{H} / \mathrm{R}$ injury in $\mathrm{H} 9 \mathrm{c} 2$ cardiomyocytes. 